\title{
Activation of the sinoaortic baroreceptor reflex arc induces analgesia: Interactions between cardiovascular and endogenous pain inhibition systems
}

\author{
ALAN RANDICH and CERIC HARTUNIAN \\ University of Iowa, Iowa City, Iowa
}

\begin{abstract}
Two experiments examined the view that activation of the sinoaortic baroreceptor reflex arc induces analgesia. Rats were instrumented with carotid artery and jugular vein cannulae for measurement of arterial blood pressure, central venous blood pressure, and heart rate during assays of pain sensitivity using tail-flick responses to radiant heat. In Experiment 1, increases in arterial blood pressure effected by infusion of phenylephrine resulted in profound analgesia that persisted for a 28-min observation period following termination of the infusion. During the phenylephrine infusion period, the degree of analgesia was significantly correlated to the degree of reflex bradycardia, but was not significantly correlated to changes in either arterial or venous blood pressure. In Experiment 2, increases in arterial blood pressure effected by infusion of phenylephrine failed to induce analgesia in rats with bilateral sinoaortic deafferentations, indicating that activation of the sinoaortic baroreceptor reflex arc is required for this form of analgesia. As in Experiment 1, reflex bradycardia, rather than increases in arterial blood pressure, was correlated with analgesia. These outcomes are consistent with the view that systems involved in the regulation of blood pressure are physiologically linked to systems involved in the regulation of pain. The implications of these findings for the etiology of hypertension are discussed.
\end{abstract}

Administration of sympathomimetic compounds induces a reduction in pain sensitivity, or analgesia, in a variety of species, including humans (Fellows \& Ullyot, 1951; Goetzl, Burrill, \& Ivy, 1944; Randall \& Selitto, 1958; Witkin, Heubner, Galdi, O'Keefe, Spitalletta, \& Plummer, 1961). For instance, subcutaneous administration of norepinephrine, methoxamine, methamphetamine, or 2-aminoindane induces analgesia in rats and mice as assayed by the inflamed foot, writhing, or hot-plate tests of pain sensitivity (Colville \& Chaplin, 1964; Little \& Rees, 1978). In general, these studies have favored the view that central, rather than peripheral, actions of sympathomimetic compounds are responsible for the analgesia, an interpretation consistent with known central adrenergic mechanisms of pain inhibition (see Harvey \& Simansky, 1981).

However, an alternative mechanism of action of some sympathomimetic compounds is suggested by recent demonstrations that genetic, renal, and DOCAsalt hypertensive rats manifest analgesia in some assays of pain sensitivity (Maixner, Touw, Brody,

This research was supported by an N.I.H. grant (NS18341) to A. Randich. We gratefully acknowledge the technical and secretarial help provided by $\mathbf{P}$. Nichols and $\mathbf{M}$. Bowersox, respectively. The authors' mailing address is: Department of Psychology, University of Iowa, Iowa City, Iowa 52242 .
Gebhart, \& Long, 1982; Randich, 1982; Randich \& Maixner, 1981; Zamir \& Segal, 1979; Zamir, Simantov, \& Segal, 1980). Specifically, it is possible that activation of the sinoaortic baroreceptor reflex arc resulting from sympathomimetic-induced increases in arterial blood pressure is capable of engaging endogenous pain inhibition systems. This view is supported indirectly by a number of experimental findings. First, peripheral administration of phenylephrine in doses capable of increasing arterial blood pressure attenuates wheel-turn escape/avoidance responding produced by aversive trigeminal stimulation in rats, but this attenuation effect does not occur following bilateral sinoaortic deafferentation (Dworkin, Filewich, Miller, Craigmyle, \& Pickering, 1979). Second, bilateral sinoaortic deafferentation lowers the threshold for both flinch and jump responses to an electric shock stimulus in spontaneously hypertensive rats (SHRs) and Wistar-Kyoto (WKYs) normotensive rats (Randich, 1982). Third, treatments that lower arterial blood pressure in hypertensive rats, for example, administration of hexamethonium in SHRs (Maixner et al., 1982) or removal of the stenotic kidney in renal hypertensive rats (Zamir \& Segal, 1979), are associated with a loss of analgesia. Finally, Maixner and Randich (Note 1) demonstrated that elevations in central venous pressure by a volume loading procedure induces a pro- 
found, long-lasting analgesia in rats. These authors argued that activation of low-pressure cardiopulmonary baroreceptors is a sufficient condition for the production of analgesia. Although this experiment does not bear directly upon the role of arterial blood pressure in modulating pain sensitivity, it is consistent with the more general proposal that systems involved in cardiovascular regulation are physiologically linked to systems modulating pain perception.

The purpose of the present experiments was to assess directly the view that increases in arterial blood pressure and concomitant activation of the sinoaortic baroreceptor reflex arc are capable of activating endogenous pain inhibition systems. In these experiments, increases in arterial blood pressure were produced by infusions of phenylephrine and pain sensitivity was assayed by tail-flick responses to radiant heat. Arterial blood pressure, central venous blood pressure, and heart rate were recorded during all phases of the experiment. Experiment 1 examined whether increases in arterial blood pressure induced analgesia in normal rats. Experiment 2 examined whether phenylephrine-induced analgesia was a consequence of arterial pressure activation of the sinoaortic baroreceptor reflex arc by assessing pain sensitivity in rats with either sham operations or bilateral sinoaortic deafferentations.

\section{EXPERIMENT 1}

In Experiment 1, rats were implanted with a right jugular vein cannula, for administration of phenylephrine and recording of central venous pressure, and a left carotid cannula, for recording of arterial blood pressure and heart rate. Phenylephrine was infused at various rates to produce graded increases in arterial blood pressure as a means of determining whether the degree of analgesia reflected the magnitude of the pressure increment. Measurements of pain sensitivity (tail-flick) to a radiant heat stimulus were obtained every $2 \mathrm{~min}$. If increases in arterial blood pressure and concomitant activation of the sinoaortic baroreceptor reflex arc induce analgesia, then the rats should show reductions in tail-flick response latencies in proportion to increases in arterial pressure.

\section{Method}

Subjects. Six male Sprague-Dawley rats obtained from Hormone Assay Laboratories in Chicago served as subjects. The rats were individually housed in wire-mesh cages in a colony room under a 12:12 h light-dark cycle. Food and water were available in the home cages on an ad-lib basis.

Apparatus. Nociceptive responses were measured with a tailflick apparatus. The radiant heat stimulus was produced by a 35$\mathrm{mm}$ projector bulb housed in a metal casing and focused on the rat's tail through a small opening in the metal housing. Onset and termination of each trial were controlled automatically by a digital timer. The intensity of the radiant heat stimulus, which was constant for all subjects, was adjusted to produce a tail-flick response of approximately 3-4 sec in a normal rat.

Arterial blood pressure, heart rate, and central venous blood pressure were recorded on a Beckman $\mathrm{R} 11 \mathrm{~A}$ rectilinear dynagraph. The arterial blood pressure signal was transduced by a Century pressure transducer, and the venous blood pressure signal was transduced by a Statham pressure transducer.

Surgical techniques. Each rat was anesthetized with Equithesin $(3 \mathrm{ml} / \mathrm{kg}$ ). An incision was made in the pectoral region $1 \mathrm{~cm}$ to the right of the midventral line and extending $2 \mathrm{~cm}$ craniad from the pectoralis. The external jugular vein was exposed at its junction with the right subclavian vein, and the connective tissue surrounding the area was cleared. A cannula (Silastic) was inserted caudad through the brachiocephalic vein approximately $4 \mathrm{~cm}$. The cannula was anchored to adjacent tissue.

A 3-cm midventral incision was then made in the cervical region. Dissection continued until the underlying sternocleidomastoid group was exposed. The left sternomastoid was displayed laterally, and the deeper omohyoid was cut at right angles to its fiber direction. The left common carotid artery was then separated from the surrounding tissues and nerves. A cannula was advanced caudad 3-4 cm and anchored to adjacent muscles (Micro-line).

The arterial and venous cannulae were then drawn subcutaneously around the neck and exited through a dorsal incision. The cannulas were anchored to the neck and flushed with a salineheparin solution.

Testing. All rats were allowed a minimum of 1 day of recovery prior to testing. Each rat was then placed in a Plexiglas restraining tube, and the cannulas were connected to the pressure transducers and an infusion pump (Harvard Apparatus 941). Saline was then infused through the venous cannula, and initial baseline tail-flick latencies were obtained every $2 \mathrm{~min}$.

Following stabilization of the tail-flick response, phenylephrine $(1 \mathrm{mg} / \mathrm{ml})$ was infused through the venous cannula at rates ranging from 24 to $192 \mu \mathrm{g} / \mathrm{min}$. Tail-flick trials were presented every $2 \mathrm{~min}$, and the infusion procedure continued until the rat exhibited a tail-flick latency of no more than $10 \mathrm{sec}$. When a 10-sec latency was obtained, the infusion procedure was stopped and tail-flick trials were administered across a 28 -min observation period. Trials were administered every $1 \mathrm{~min}$ during the first $10 \mathrm{~min}$ of the observation period and every $3 \mathrm{~min}$ during the remaining $18 \mathrm{~min}$ of the observation period. Arterial blood pressure, central venous blood pressure, and heart rate were recorded simultaneously during all tail-flick trials.

Data analysis. Linear regression was used to analyze the data. Alpha was set at 0.05 in all analyses.

\section{Results}

Arterial blood pressure, heart rate, and venous blood pressure were recorded during the infusions of phenylephrine and tail-flick trials. The changes in each of these measures from the initial baseline values obtained during the infusions of saline were compared with changes in tail-flick latencies from their initial baseline values. The results of these correlational analyses indicated that (1) there was no signifcant correlation between increases in tail-flick latencies from baseline and increases in arterial blood pressure $[r=.13 ; F(1,61)=1.05]$, (2) there was a significant correlation between increases in tail-flick latencies and decreases in heart rate $[r=.25 ; F(1,61)$ $=3.35$ ], and (3) there was no significant correlation between increases in tail-flick latencies and increases in venous pressure $[r=.07 ; F(1,60)=0.34]$. A separate analysis of variance on the integrity of the baroreceptor reflex arc indicated a significant correlation 
between decreases in heart rate and increases in arterial blood pressure $[r=.77 ; F(1,61)=73.79]$.

Infusions of phenylephrine in two of the six rats were terminated in midcourse because of loss of a signal. The remaining four rats all achieved the maximum response of a 10 -sec tail-flick latency indicating profound analgesia. At this point, the infusion procedure was terminated and tail-flick latencies and cardiovascular variables were recorded during the 28min observation period. Figure 1 presents changes in mean tail-flick latencies (absolute values) and mean
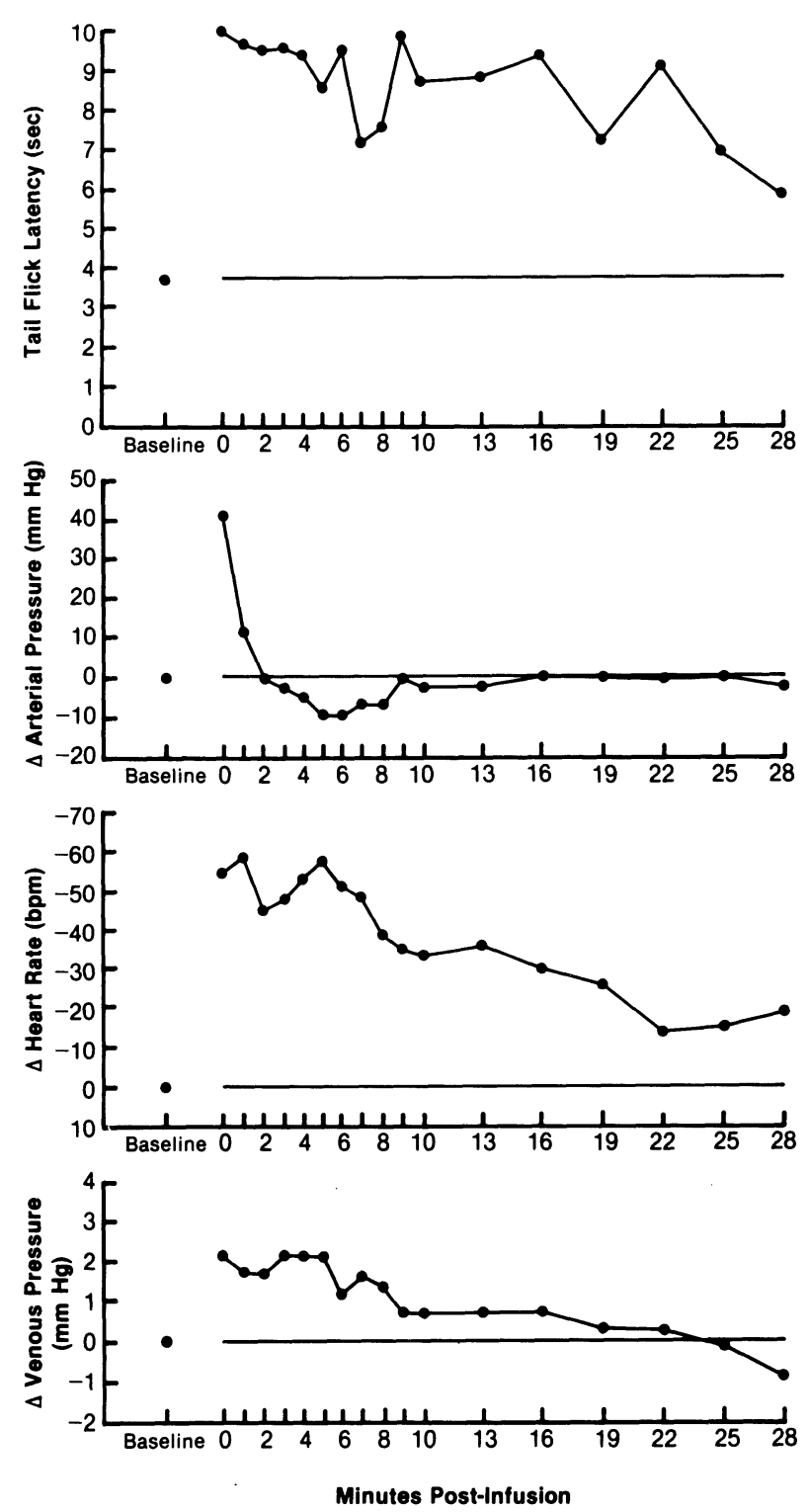

Figure 1. Mean tail-flick latencies (absolute values), mean changes in arterial blood pressure from baseline, mean changes in heart rate from baseline, and mean changes in central venous blood pressure from baseline during the 28 -min observation period following termination of the infusion procedure. Time point zero represents the first occurrence of a 10-sec tail-flick latency. cardiovascular responses (changes from baseline) during this observation period. The top panel of Figure 1 shows that mean tail-flick latencies remained elevated above baseline values during the entire 28min observation period. A randomized block analysis of variance also indicated that there were no significant reductions in tail-flick latencies during the observation period $[\mathrm{F}(1,46)=1.81]$, although there was a trend towards a decrease in tail-flick latencies with time. The second panel of Figure 1 shows that mean arterial blood pressure rapidly returned to baseline or below baseline values following termination of phenylephrine infusion before stabilizing. There was a significant decrease in arterial blood pressure during the observation period $[F(1,46)=$ 6.18]. The third panel of Figure 1 shows that mean heart rate did tend to normalize following termination of phenylephrine infusion but that, similar to tail-flick responses, there were no significant changes in heart rate during the 28 -min observation period $[F(1,40)=1.19]$. Thus, both increases in tail-flick latencies and bradycardia far outlast changes in arterial pressure. Finally, the bottom panel of Figure 1 indicates that there were only small increases in venous pressure produced by phenylephrine, and a randomized block analysis of variance indicated no significant changes in venous pressure during the observation period $[\mathrm{F}(1,46)=1.72]$.

\section{Discussion}

Experiment 1 demonstrates that administration of phenylephrine produces a profound, long-lasting analgesia, as indexed by an increase in tail-flick latencies to radiant heat. Only the magnitude of reflex bradycardia was significantly related to increases in tail-flick latencies during infusions of phenylephrine. However, this outcome is to be expected, since reflex bradycardia is one of the correctional mechanisms for the regulated system variable of arterial blood pressure. Hence, reflex bradycardia should provide a good index of the degree of activation of the sinoaortic baroreceptor reflex arc rather than arterial pressure per se.

The analgesia persisted unabated during a 28-min observation period following termination of phenylephrine infusion. During this time, arterial blood pressure rapidly returned to normal or below normal values, whereas bradycardia was sustained. There were few or no changes in venous pressure during the phenylephrine infusion or following termination of the infusion, indicating that increases in venous pressure were unlikely to contribute to the analgesic response (see Maixner \& Randich, 1983). Thus, the present outcomes are consistent with the hypothesis that elevations in arterial blood pressure are capable of inducing analgesia. However, the present study does not rule out the possibility that phenylephrine was acting centrally to produce analgesia. Although 
phenylephrine does not readily cross the blood-brain barrier, the pressure increases effected by phenylephrine in this experiment could be assumed to be sufficient to break down the barrier and permit both entry and a central effect of phenylephrine.

\section{EXPERIMENT 2}

The purpose of Experiment 2 was to determine whether an increase in arterial blood pressure and concomitant activation of the sinoaortic baroreceptor reflex arc was the actual stimulus for the analgesia induced by infusion of phenylephrine. This was accomplished by assaying pain sensitivity in rats with either sham operations or bilateral sinoaortic deafferentations (SADs). The bilateral SAD eliminates afferents from the sinoaortic baroreceptors, conveying information about arterial blood pressure. Thus, rats with bilateral SADs should fail to show any analgesia following infusions of phenylephrine if the stimulus is an increase in arterial blood pressure.

\section{Method}

Subjects. Ten male Sprague-Dawley rats obtained from Hormone Assay Laboratories in Chicago served as subjects. All other conditions were as described in Experiment 1.

Apparatus. The apparatus was as described in Experiment 1.

Surgical techniques. The surgical techniques for implantation of cannulas were as reported in Experiment 1 . In addition, five rats received bilateral SADs according to the method of Krieger (1964). Briefly, the vagus and carotid artery were separated from the sympathetic trunks and neurovascular sheath. The superior laryngeal nerves were resected and the sympathetic trunks were resected caudal to the superior cervical ganglion. Denervation of the carotid sinus baroreceptors was accomplished by stripping the bifurcation of all fibers and then painting the region with a solution of $10 \%$ phenol in ethanol. The integrity of the SAD was confirmed during subsequent infusions of phenylephrine, which failed to produce reflex bradycardia.

Testing. All rats received essentially the same testing procedure described in Experiment 1, with the following modifications. First, the rate of phenylephrine infusion was held constant at $24 \mu \mathrm{g} / \mathrm{min}$. This change in procedure was adopted to maintain steady state activation of the sinoaortic baroreceptor reflex arc. Second, the infusion period was fixed at $22 \mathrm{~min}$. Third, only a 10-min observation period was used following termination of the infusion, with a tail-flick trial being administered each minute.

\section{Results}

All five of the sham-operated rats showed increases in tail-flick latencies during infusions of phenylephrine and attained the 10-sec criterion latency within the 22-min infusion period. None of the five rats with bilateral SADs showed a 10-sec tail-flick latency during the 22-min infusion period or any consistent increases in tail-flick latencies. Figure 2 presents scatter plots of tail-flick latencies (changes from baseline values) as a function of changes in arterial pressure (top panel), heart rate (middle panel), and venous pressure (bottom panel) in both sham-operated and SAD rats during the infusions of phenylephrine. A least squares linear regression function is also plotted in each panel for descriptive purposes.
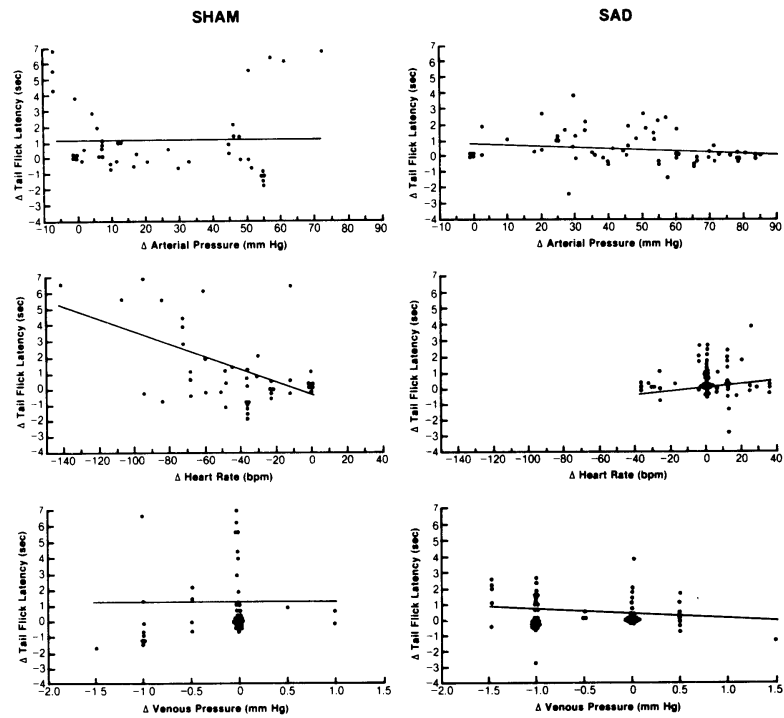

Figure 2. Scatter plots and least squares regression functions for changes in tail-flick latencies during infusions of phenylephrine as a function of changes in arterial blood pressure (top panels), changes in heart rate (middle panels), and changes in central venous blood pressure (bottom panels) in sham-operated rats (left panels) and in rats with bilateral SADs (right panels).

In sham-operated rats, increases in tail-flick latencies during infusions of phenylephrine were (1) not significantly correlated with increases in arterial blood pressure $[r=.00 ; F(1,44)=0.00]$, (2) significantly correlated with decreases in heart rate $[r=.53$; $F(1,44)=17.42]$, and (3) not significantly correlated with increases in venous pressure $[r=.00 ; F(1,44)$ $=0.00$ ]. Thus, as reported in Experiment 1 , the degree of analgesia is related only to the degree of reflex bradycardia induced by the increase in arterial pressure. A similar analysis of responses in SADs is shown in the right panels of Figure 2. Specifically, increases in tail-flick latencies during the infusion period were not significantly correlated with (1) increases in arterial blood pressure $[\mathrm{r}=.21 ; \mathrm{F}(1,62)=2.86]$, (2) decreases in heart rate $[\mathrm{r}=.00 ; \mathrm{F}(1,62)=0.08]$, or $(3)$ increases in venous pressure $[r=.16 ; F(1,62)=1.79]$. Thus, bilateral sinoaortic deafferentation eliminated the action of phenylephrine in inducing analgesia. It should be noted that the middle panel also demonstrates the efficacy of the bilateral SAD. Although mild bradycardia was observed in some rats, this was not associated with pressure increases.

Figure 3 presents mean tail-flick latencies (absolute values) during the 10 -min observation period following termination of phenylephrine infusion in shamoperated rats and corresponding mean cardiovascular responses. The outcomes were similar to those reported during the initial $10 \mathrm{~min}$ of the $28-\mathrm{min}$ observation period used in Experiment 1. There was a profound, long-lasting analgesia that did not significantly decrease during the observation period $[F(1,40)$ 


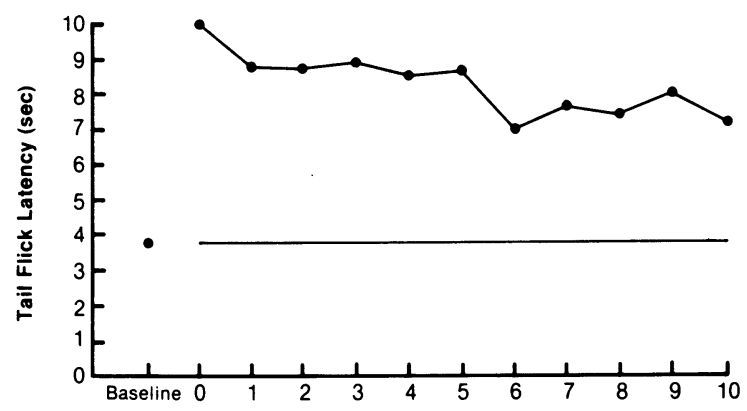

concomitant activation of the sinoaortic baroreceptor arc, and neither a central effect of phenylephrine nor some nonspecific drug effect.

As noted in Experiment 1, the degree of analgesia during phenylephrine infusion was significantly correlated with reflex bradycardia and not with increased arterial pressure. Indeed, using a low constant rate of infusion made it possible for some sham-operated rats to regulate arterial blood pressure at a near normal value through steady decreases in heart rate.

Finally, sham-operated rats all manifested profound analgesia similar to that reported in Experiment 1 . The analgesia persisted in an undiminished form during the 10-min observation period, while arterial blood pressure rapidly returned towards baseline values. Only the bradycardia response was sustained during the 10 -min observation period. The implications of this will be discussed later.

\section{GENERAL DISCUSSION}

The experiments demonstrate that activation of the sinoaortic baroreceptor reflex arc (as indexed by elicitation of reflex bradycardia) by increases in arterial blood pressure is capable of inducing analgesia, as assayed by the tail-flick response to painful radiant heat. That the stimulus for the analgesia was an increase in arterial blood pressure rather than either a central or a nonspecific effect of phenylephrine was demonstrated by the failure of rats with bilateral SADs to show any consistent increases in tail-flick latencies with increases in arterial blood pressure, when the magnitude of the pressure increases were comparable to or exceeded those induced in shamoperated rats. However, it is important to note that a 22-min infusion period was used in Experiment 2. There is always the possibility that analgesia may have been induced in rats with bilateral SADs with longer infusion periods, for example, $1 \mathrm{~h}$, but this possibility seems unlikely given that all sham-operated rats showed analgesia within the 22-min period.

The degree of analgesia during infusions of phenylephrine was significantly correlated with the magnitude of reflex bradycardia in Experiment $2(r=0.53)$, but was not significantly correlated with either increases in arterial blood pressure or increases in central venous blood pressure. Several aspects of these relationships deserve consideration. First, arterial blood pressure is determined by both cardiac output and total peripheral resistance. In this sense, arterial blood pressure should be viewed as the system variable that is regulated by the correctional mechanisms of changes in both heart rate and sympathetic tone. Thus, the best index for the degree of activation of the sinoaortic baroreceptor reflex arc would be the extent to which these correctional mechanisms are engaged rather than changes in arterial blood pressure per se. Indeed, several sham-operated rats in 
Experiment 2 showed elevations in tail-flick latencies while maintaining arterial blood pressure at near normal values during infusions of phenylephrine. However, the baroreceptor reflex arc appeared to be engaged, since steady decreases in heart rate were observed. Thus, we would expect arterial blood pressure to be related to the degree of analgesia only when these correctional mechanisms are fully engaged but failing to adequately lower arterial blood pressure.

There were several instances in which tail-flick latencies were not elevated despite profound hypertension and bradycardia. This indicates that factors other than simple activation of the sinoaortic baroreceptor reflex arc contribute to the production of the analgesia. Factors such as the total peripheral resistance, the rate of arterial pressure increase, baroreceptor gain, baroreceptor threshold, and the length of time arterial pressure is elevated should be considered. Moreover, the analgesic response was maintained over a 28-min observation period following termination of the infusion of phenylephrine and at a time when arterial blood pressure was at normal or below normal values. Thus, it is plausible to assume that activation of the sinoaortic baroreceptor reflex arc may "trigger" the release of some longlasting peptide or hormone to produce this analgesia.

The present experiments did not address the involvement of opioids, but it is possible that the "read" for the analgesic response is in some fashion related to structures subserving the opioid-modulated control of either vagal or sympathetic tone, since bradycardia is often associated with analgesia. The administration of morphine-like agents and opioid peptides have been shown to increase vagal tone, reduce sympathetic tone, and induce a long-lasting analgesia. Possible sites of action of these agents include the nucleus of the solitary tract, nucleus gigantocellularis reticularis, and nucleus ambiguus (Chan \& Kuo, 1980; Chen \& Chan, 1980; Feldberg \& Wei, 1977; Florez \& Mediavilla, 1977; Laubie \& Schmitt, 1981; Laubie, Schmitt, Canellas, Roquebert, \& Desmichel, 1974; Lemaire, Tseng, \& Lemaire, 1978).

There are also interesting parallels between the outcomes of the present experiments and those of Maixner and Randich (Note 1). In the latter experiment, analgesia was induced by a volume-loading procedure in which Ficoll (a volume expander) was infused into the right jugular vein. This volumeloading procedure resulted in an increase in central venous pressure, reflex bradycardia, mild hypotension, and analgesia as assayed by the tail-flick response. The magnitude and duration of both the analgesia and reflex bradycardia were similar to those observed in the present experiments. Maixner and Randich (Note 1) argued that the effective stimulus for the analgesia obtained with the volumeloading procedure was activation of vagal afferents, since resection of the right vagal nerve trunk not only attenuated the analgesic response, but also eliminated the correlation between increases in venous pressure and the magnitude of analgesia. Collectively, these findings suggest that endogenous pain inhibition systems receive multiple inputs from cardiovascular regulatory mechanisms, although it is unclear whether low-pressure vagal afferents and high-pressure sinoaortic afferents converge on the same neuronal pool to induce analgesia.

Finally, it is important to consider whether alterations in pain sensitivity are important for the etiology of hypertension. Other studies (Maixner et al., 1982; Randich, 1982) have shown that resection of the right vagal nerve trunk but not bilateral SAD eliminates the analgesia manifested by SHRs in the hot-plate assay of pain sensitivity. Although these outcomes suggest that changes in central venous pressure rather than arterial blood pressure are critical for the analgesia manifested by SHRs, the bilateral SADs were carried out after the development of hypertension. Thus, it is possible that initial increases in arterial blood pressure during the development of hypertension contribute to the production of the analgesia through the mechanisms elucidated in the present experiments.

On the other hand, it is useful to consider whether interactions between systems involved in the regulation of blood pressure and endogenous pain inhibition are important for the etiology of hypertension. In this view, increases in either arterial or venous blood pressure could provide a short-term active coping response to stress, since reductions in pain sensitivity and possible associated motivational effects, for example, sedation, would represent some form of psychophysiological reflief from stress. Engaging these mechanisms might serve to reinforce the increase in blood pressure through conditioning and learning mechanisms, and make them more likely to occur either in advance of or under similar stimulus situations in the future. Thus, an animal may learn to increase blood pressure as a means of coping with stress, but this eventuates in sustained hypertension leading to deleterious cardiovascular functioning.

\section{REFERENCE NOTE}

1. Maixner, W., \& Randich, A. The role of the right vagal nerve trunk in antinociception. Manuscript submitted for publication, 1983.

\section{REFERENCES}

Chan, S. H. H., \& Kuo, J. S. Interaction of gigantocellular reticular nucleus with reflex bradycardia and tachycardia in the cat. Brain Research, 1980, 182, 457-460.

Chen, Y. H., \& Chan, S. H. H. The involvement of gigantocellular reticular nucleus in clonidine-promoted hypotension and bradycardia in experimentally-induced hypertensive cats. Neuropharmacology, 1980, 19, 939-945. 
Colville, K. I., \& Chaplin, E. Sympathomimetics as analgesics: Effects of methoxamine, methamphetamine, metaraminol, and norepinephrine. Life Sciences, 1964, 3, 315-322.

Dworkin, B. R., Filewich, R. J., Miller, N. E., Craigmyle, N., \& Pickering, T. G. Baroreceptor activation reduces reactivity to noxious stimulation: Implications for hypertension. Science, 1979, 205, 1299-1301.

Feldberg, W., \& Wei, E. The central origin and mechanism of cardiovascular effects of morphine as revealed by naloxone in cats. Journal of Physiology (London), 1977, 272, 99-100.

Fellows, E. J., \& Ullyot, G. E. Analgesics: A. Aralkylamines. In C. M. Suter (Ed.), Medicinal chemistry. New York: Wiley, 1951.

Florez, J., \& Mediavilla, A. Respiratory and cardiovascular effects of metenkephalin applied to the ventral surface of the brainstem. Brain Research, 1977, 138, 585-590.

Goetzl, F. R., Burrill, D. Y., \& Ivy, A. C. The analgesic effect of morphine alone and in combination with dextroamphetamine. Proceedings of the Society of Experimental Biology and Medicine, 1944, 55, 248-250.

Harvey, J. A., \& Simansky, K. J. The role of serotonin in modulation of nociceptive reflexes. In B. Harber (Ed.), Serotonin: Current aspects of neurochemistry and function. New York: Plenum Press, 1981.

KrIEger, E. M. Neurogenic hypertension in the rat. Circulation Research, 1964, 15, 511-521.

LAUBie, M., \& SchmitT, H. Sites of the vagally mediated bradycardia induced by morphine-like agents and opioid peptides. In J. P. Buckley, C. M. Ferrario, \& M. F. Lokhandwala (Eds.), Perspectives in cardiovascular research: Central nervous system mechanisms in hypertension (Vol. 6). New York: Raven Press, 1981.

Laubie, M., Schmitt, H., Canellas, J., Roquebert, J., \& Desmichel, P. Centrally mediated bradycardia and hypotension induced by narcotic analgesics: Dextromoramide and fentanyl. European Journal of Pharmacology, 1974, 28, 66-75.
Lemaire, I., Tseng, R., \& Lemaire, S. Systemic administration of B-endorphin: Potent hypotensive effect involving a serotonergic pathway. Proceedings of the National Academy of Science, 1978, 75, 6240-6242.

Little, H. J., \& REes, J. M. H. Naloxone antagonism of sympathomimetic analgesia. In J. M. Van Ree \& L. Terenius (Eds.), Characteristics and functions of opioids. Amsterdam: ElsevierNorth Holland Biomedical Press, 1978.

Maixner, W., Touw, K. B., Brody, M. J., Gebhart, G. F., \& LoNG, J. P. Factors influencing the altered pain perception in the spontaneously hypertensive rat. Brain Research, 1982, 237, 137-145.

Randall, L. O., \& Selitto, J. J. Anti-inflammatory effect of Romilar CF. Journal of the American Pharmacological Association, 1958, 47, 313-314.

RANDICH, A. Sinoaortic baroreceptor reflex arc modulation of nociception in spontaneously hypertensive and normotensive rats. Physiological Psychology, 1982, 10, 267-272.

Randich, A., \& Maixner, W. Acquisition of conditioned suppression and responsivity to thermal stimulation in spontaneously hypertensive, renal hypertensive and normotensive rats. Physiology \& Behavior, 1981, 27, 585-590.

Witkin, L. B., Heubner, C. F., Galdi, F., O'Keefe, E., Spitalletta, P., \& Plummer, A. J. Pharmacology of 2amino-indane hydochloride (SU-8629): A potent non-narcotic analgesic. Journal of Pharmacology and Experimental Therapeutics, 1961, 133, 400-408.

ZAmir, N., \& SEgAL, M. Hypertension induced analgesia: Changes in pain sensitivity in experimental hypertensive rats. Brain Research, 1979, 201, 170-173.

Zamir, N., Simantov, R., \& Segal, M. Pain sensitivity and opioid activity in genetically and experimentally hypertensive rats. Brain Research, 1980, 184, 299-310.

(Manuscript received April 11, 1983; revision accepted for publication July $25,1983$. ) 